Typeset with jpsj2.cls $<$ ver.1.2 $>$

FULl PAPER

\title{
Effect of Fermi Surface Topology on Inter-Layer Magnetoresistance in Layered Multiband Systems: Application to $\mathrm{LaFeAsO}_{1-x} \mathrm{~F}_{x}$
}

\author{
Takao Morinari ${ }^{1 *}$, Hiroki NakAmura ${ }^{2,3,4}$, Masahiko MachidA ${ }^{2,3,4}$, and Takami TohyAmA ${ }^{1,4}$ \\ ${ }^{1}$ Yukawa Institute for Theoretical Physics, Kyoto University, Kyoto 606-8502, Japan \\ ${ }^{2}$ CCSE, Japan Atomic Energy Agency, 6-9-3 Higashi-Ueno, Tokyo 110-0015, Japan \\ ${ }^{3}$ CREST(JST), 4-1-8 Honcho, Kawaguchi, Saitama 332-0012, Japan \\ ${ }^{4}$ JST, Transformative Research-Project on Iron Pnictides (TRIP), Chiyoda, Tokyo 102-0075, Japan
}

\begin{abstract}
In layered single band systems, the interlayer conductivity depends on the orientation of the in-plane magnetic field and takes maximum values when the magnetic field is perpendicular to flat regions of the Fermi surface. Extending this known results to multi-band systems, we propose an experiment to extract information about their Fermi surface topology. We discuss application of the formula to a FeAs-based superconductor, $\mathrm{LaFeAsO}_{1-x} \mathrm{~F}_{x}$. We show that the magnetically ordered state in the parent compound is clearly distinguished from the paramagnetic state by the oscillation period in the interlayer conductivity. We demonstrate that evolution of the Fermi surface topology by changing the doping concentration is reflected to the interlayer conductivity oscillation patterns.
\end{abstract}

KEYWORDS: Fermi surface, interlayer magnetoresistance, Fe-pnictide

\section{Introduction}

Determination of the Fermi surface topology plays an important role to make clear the basic electronic structure of a superconductor. For layered compounds, angle-resolved photoemission spectroscopy (ARPES) can be used to study the Fermi surface. Although ARPES provides the map of the Fermi surface, ARPES measurements are sensitive to surface conditions. Contrary, magnetic quantum oscillations provide information about the Fermi surface area perpendicular to the applied magnetic field. The Fermi surface areas determined by magnetic quantum oscillations are bulk electronic properties but this technique does not provide information about the Fermi surface topology directly. In this paper, we propose interlayer magnetoresistance experiments to probe the bulk Fermi surface topology in layered multi-band systems and its application to the newly discovered FeAs-based layered superconductors. ${ }^{1}$ To be specific, we focus on $\mathrm{LaFeAsO}_{1-x} \mathrm{~F}_{x}$ and discuss what we expect for the interlayer magnetoresistance in the paramagnetic state and the magnetically ordered state.

The Fermi surfaces of the FeAs-based layered superconductors have been studied theoretically and experimentally. First principles calculations ${ }^{2,3}$ suggest that the Fermi surface of LaFeAsO consists of hole Fermi surfaces around $\Gamma$ point and electron Fermi surfaces around $M$ point. The Fermi surfaces measured

*morinari@yukawa.kyoto-u.ac.jp 
J. Phys. Soc. Jpn.

Full Paper

by $\mathrm{ARPES}^{4-8}$ are mostly consistent with this picture, though the measured samples are limited. For example, a single hole pocket and a single electron pocket were observed in $\mathrm{NdFeAsO}_{0.9} \mathrm{~F}_{0.1} \cdot{ }^{8,9}$ while the other Fermi pockets predicted by the band calculations were not observed. The hole Fermi surfaces and the electron Fermi surfaces have instability of a magnetic order associated with a nesting of $(\pi, \pi)$ wavevector. The magnetically ordered state is a collinear antiferromagnetic state as suggested from the neutron scattering experiments. ${ }^{10,11}$ Quantum oscillation measurements were carried out for magnetic states of a few Fe-based superconductors. ${ }^{12-14}$ Possible folding of Brillouin zone are discussed so that the observed oscillation periods are reproduced.

Another experiment to probe the Fermi surface of layered compounds is to measure angular magnetoresistance oscillations which was firstly discovered in the organic conductors $\beta$-(BEDT-TTF $)_{2} \mathrm{IBr}_{2}{ }^{15}$ and $\theta$-(BEDT-TTF $)_{2} \mathrm{I}_{3} \cdot{ }^{16}$ The magnetoresistance oscillations in these systems were well understood by a semiclassical analysis assuming an energy dispersion described by a two-dimensional cylinder with a weak cosine warping. ${ }^{17,18}$ In this magnetoresistance phenomenon, if the applied magnetic field lies in the plane, ${ }^{19-21}$ the oscillation patterns are associated with the Fermi surface topology. The interlayer conductivity is dominated by the regions of the Fermi surface where the Fermi velocity is parallel to the magnetic field. The theoretical calculation for $\mathrm{Tl}_{2} \mathrm{Ba}_{2} \mathrm{CuO}_{6}{ }^{21}$ is consistent with the experiment. ${ }^{22}$

In this paper, we extend the formula for the single-band systems ${ }^{19-21}$ to multi-band systems. The derivation is given in Sec.2. We apply the formula to the paramagnetic state and the magnetic state of $\mathrm{LaFeAsO}$ (Sec.3) and the paramagnetic state of $\mathrm{LaFeAsO}_{1-x} \mathrm{~F}_{x}$ (Sec.4). The electronic band structure of the parent compound is determined by first principles calculations. For doped systems, we use the five band model described in ref.23. We consider $\mathrm{LaFeAsO}_{1-x} \mathrm{~F}_{x}$ because two-dimensionality is stronger than other FeAs-based superconductors, such as $\mathrm{BaFe}_{2} \mathrm{As}_{2}$.

\section{Interlayer Conductivity Formula}

In this section, we derive the angular magnetoresistance formula for multi-band systems. We assume that the system has a structure of stacking conduction layers in the $z$ axis. The magnetic field $\mathbf{B}=$ $\left(B_{x}, B_{y}, 0\right)=B(\cos \phi, \sin \phi, 0)$ is assumed to be in the plane. The Fermi surface topology is associated with the azimuthal angle $\phi$ dependence of the interlayer magnetoresistance as we shall see below. The vector potential $\mathbf{A}$ is taken as $\mathbf{A}=\left(B_{y} z,-B_{x} z, 0\right)$.

The interlayer tunneling between $j$-th plane and $j+1$-th plane is described by

$$
H_{t}=-t_{c} \sum_{j, \nu, \sigma=\uparrow, \downarrow} \int d^{2} \mathbf{r} \psi_{j+1, \sigma}^{(\nu) \dagger}(\mathbf{r}) \psi_{j, \sigma}^{(\nu)}(\mathbf{r}) \exp (i \chi(\mathbf{r}))+h . c .,
$$

where $\nu$ represents the band index. Here we have included the effect of the in-plane magnetic field via a Pierls procedure:

$$
\chi(\mathbf{r})=\frac{e}{c \hbar} a_{c}\left(B_{y} x-B_{x} y\right)
$$

with $a_{c}$ the interlayer spacing along the $z$ axis. The interlayer conductivity is calculated by the Kubo 
formula, ${ }^{24}$

$$
\sigma_{z z}=\lim _{\omega \rightarrow 0} \frac{\operatorname{Im} K_{z z}^{R}(\omega)}{\omega}
$$

where $K_{z z}^{R}(\omega)$ is the Fourier transform of the following retarded current-current correlation function,

$$
K_{z z}^{R}\left(t-t^{\prime}\right)=-\frac{i}{\hbar S} \theta\left(t-t^{\prime}\right)\left\langle T_{\tau}\left[J_{z}(t), J_{z}\left(t^{\prime}\right)\right]\right\rangle
$$

with $S$ the area of the system. We calculate $K_{z z}^{R}(t)$ in the Matsubara formalism. The correlation function in the Matsubara formalism is given by

$$
K_{z z}^{M}(\tau)=\frac{1}{S}\left\langle T_{\tau} J_{z}(\tau) J_{z}(0)\right\rangle
$$

Here the current operator is

$$
J_{z}=\frac{i e a_{c} t_{c}}{\hbar} \sum_{j, \nu, \sigma} \int d^{2} \mathbf{r} \psi_{j+1, \sigma}^{(\nu) \dagger}(\mathbf{r}) \psi_{j, \sigma}^{(\nu)}(\mathbf{r}) \exp (i \chi(\mathbf{r}))+h . c .
$$

The function $K_{z z}^{R}(\omega)$ is obtained by $K_{z z}^{R}(\omega)=K_{z z}^{M}\left(i \omega_{n} \rightarrow \omega+i \delta\right)$ where $K_{z z}^{M}\left(i \omega_{n}\right)=$ $\int_{0}^{\beta} d \tau \exp \left(i \omega_{n} \tau\right) K_{z z}^{M}(\tau)$ and $\delta$ is a positive infinitesimal number. For the non-interacting case, we obtain

$$
\begin{aligned}
\sigma_{z z}= & \frac{2 \pi \hbar}{S} \sum_{\mathbf{k}, \mathbf{k}^{\prime}, \nu, j, \sigma} \int_{-\infty}^{\infty} d E\left(-\frac{\partial f}{\partial E}\right) \delta\left(E-E_{\mathbf{k}, \sigma}^{(\nu)}\right) \delta\left(E_{\mathbf{k}, \sigma}^{(\nu)}-E_{\mathbf{k}^{\prime}, \sigma}^{(\nu)}\right) \\
& \times\left|\left\langle\mathbf{k}^{\prime}, j+1, \sigma\left|J_{z}\right| \mathbf{k}, j, \sigma\right\rangle\right|^{2}
\end{aligned}
$$

where the $\nu$-th energy band is denoted by $E_{\mathbf{k}}^{(\nu)}$. Substituting the matrix element of the current operaotr $J_{z}$ for the electron hopping from the $j$-th layer to the $j+1$-th layer,

$$
\left\langle\mathbf{k}^{\prime}, j+1, \sigma\left|J_{z}\right| \mathbf{k}, j, \sigma\right\rangle=\frac{i e a_{c} t_{c}}{\hbar} \delta_{\mathbf{k}, \mathbf{k}^{\prime}-\mathbf{b}}
$$

with $\mathbf{b}=e a_{c}\left(-B_{y}, B_{x}\right) /(c \hbar)$, we obtain

$$
\begin{aligned}
\sigma_{z z}= & \frac{2 e^{2} t_{c}^{2} a_{c}^{2}}{\hbar} N_{z} \sum_{\nu, \sigma} \int \frac{d^{2} \mathbf{k}}{(2 \pi)^{2}} \int_{-\infty}^{\infty} d E\left(-\frac{\partial f}{\partial E}\right) \\
& \times \delta\left(E-E_{\mathbf{k}, \sigma}^{(\nu)}\right) \frac{\Gamma}{\left(E_{\mathbf{k}, \sigma}^{(\nu)}-E_{\mathbf{k}-\mathbf{b}, \sigma}^{(\nu)}\right)^{2}+\Gamma^{2}}
\end{aligned}
$$

Here we have replaced the second Dirac delta function in eq.(7) by a Lorentz function with $\Gamma=\hbar /(2 \tau)$ to include the scattering effect upon hopping between layers. Although it is possible to take different $\Gamma$ 's for each band, we take the same $\Gamma$ for all bands for simplicity. The formula (9) is understood in a following way. According to the semiclassical equations under a magnetic field, an electron moves in $\mathbf{k}$-space upon hopping between one layer to the adjacent layers. The contribution to $\sigma_{z z}$ is large if the motion in $\mathbf{k}$-space is along the Fermi surface because the Lorentz factor in eq.(9) takes the maximum value. Since the direction of the motion is perpendicular to the in-plane magnetic field, dominant contributions arise if the magnetic field is perpendicular to the flat regions of the Fermi surface. 
J. Phys. Soc. Jpn.

Full PAPeR

At zero temperature, the integration over the Brillouin zone is transformed to the integration along the Fermi surfaces as follows

$$
\begin{aligned}
\sigma_{z z}= & \frac{e^{2}}{2 \pi}\left(\frac{t_{c} a_{c}}{\hbar}\right)^{2} N_{z} \sum_{\nu} \int_{E_{\mathbf{k}}^{(\nu)}=E_{F}} d \ell_{\mathbf{k}} \frac{1}{\left|\mathbf{v}_{\mathbf{k}}^{(\nu)}\right|} \\
& \times \frac{\Gamma / \pi}{\left(\frac{e}{c} a_{c} \mathbf{v}_{\mathbf{k}}^{(\nu)} \times \mathbf{B}\right)^{2}+\Gamma^{2}}
\end{aligned}
$$

where $\mathbf{v}_{\mathbf{k}}^{(\nu)}=(1 / \hbar) \partial E_{\mathbf{k}}^{(\nu)} / \partial \mathbf{k}$. From the form of the Lorentz factor, it is clear that dominant contributions to $\sigma_{z z}$ arise from the Fermi surface parts with the Fermi velocity being parallel to the magnetic field, which is consistent with the intuitive picture mentioned above. The weight of the contribution is determined by the factor $1 /\left|\mathbf{v}_{\mathbf{k}}^{(\nu)}\right|$ as in the density of states. Anisotropy in $\sigma_{z z}$ detects deviation of the Fermi surface shape from the circular shape. If a Fermi surface is completely circular, such a Fermi surface contributes to $\sigma_{z z}$ only as the constant value independent of the orientation of the magnetic field. In order to calculate $\sigma_{z z}$ from eq.(10) we need values of $\mathbf{v}_{\mathbf{k}}^{(\nu)}$ and the Fermi surface data for each band. For Fermi surfaces which are not given by separated lines, one needs to calculate eq.(9).

Note that we have neglected the Zeeman energy contribution in eq.(10). To be precise, we need the energy contour at $E^{( \pm)}=E_{F} \pm g \mu_{B} B$. However, the Zeeman energy is much less than the Fermi energy. Therefore, we may neglect the Fermi surface change associated with the Fermi energy shift by the Zeeman energy.

\section{Interlayer Conductivity of LaFeAsO}

For the application to $\mathrm{LaFeAsO}$, we compute the electronic band structure by first principles calculations. The Fermi surfaces were obtained by the first-principles density functional calculation package VASP. ${ }^{25}$ Figure 1(a) shows the Fermi surface of the paramagnetic state and Fig.1(b) shows the interlayer conductivity for $\Gamma=2 \times 10^{-3}$ and $B=10 \mathrm{~T}$ which corresponds to $\omega_{c} \tau=e B \tau /(m c)=0.29$. (Hereafter we measure $\Gamma$ in units of eV.) Each band contribution to the interlayer conductivity is also shown. The values are normalized by the total interlayer conductivity at $\phi=0^{\circ}$. The broad peaks in the interlayer conductivity at $\phi=45^{\circ}$ and $\phi=135^{\circ}$ are associated with the contributions from the Fermi surfaces FS3 and FS5 because those Fermi surfaces have flat regions whose Fermi velocities are in these directions. The peak at $\phi=90^{\circ}$ is associated with the flat region in the Fermi surface FS4. The Fermi surfaces FS1 and FS2 are nearly circular. Therefore, their interlayer conductivities are almost featureless. The largest contribution to $\sigma_{z z}$ comes from FS2 but other band features are clearly seen because anisotropy in the FS2 contribution is small.

The Fermi surface and the interlayer conductivity in the magnetically ordered state is shown in Figs.2(a) and 2(b). The magnetic ordered state is a collinear antiferromagnetic order. ${ }^{3}$ The interlayer conductivity is dominated by the contribution from the Fermi surface around the $\Gamma$ point (FS1). The broad peak at $\phi=90^{\circ}$ is associated with the flat regions in FS1. The small Fermi surfaces FS2 and FS3 are almost featureless because they are nearly circular. 


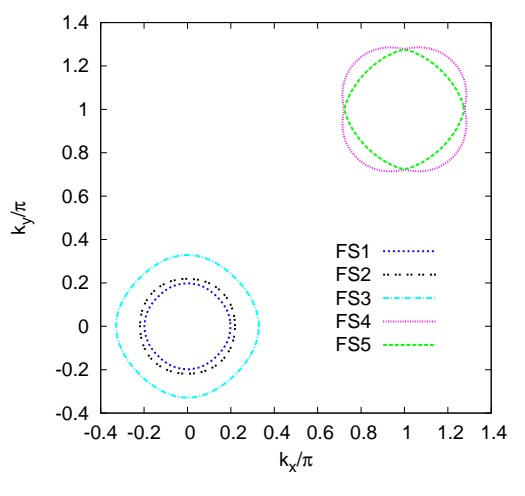

(a)

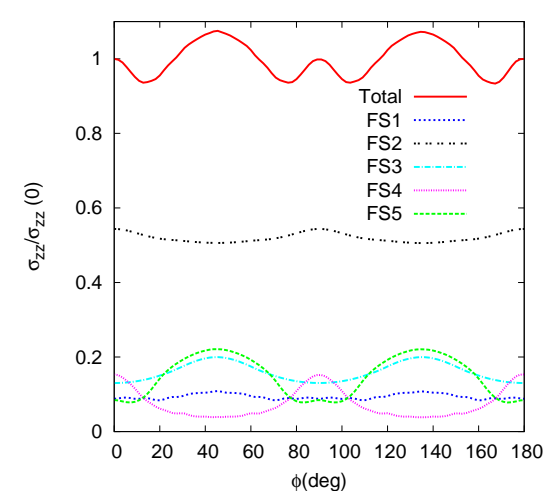

(b)

Fig. 1. (Color online) The paramagnetic state Fermi surface (a) and the normalized interlayer conductivity(b).

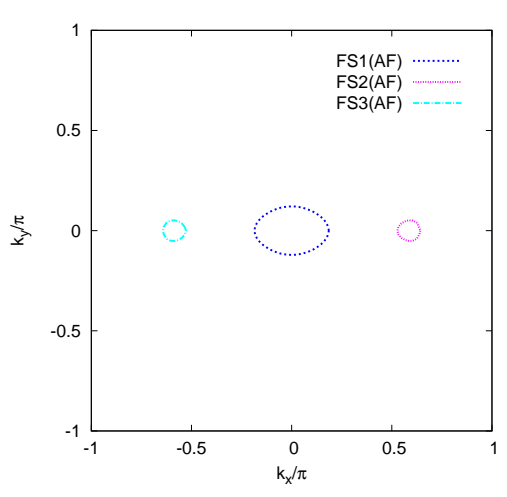

(a)

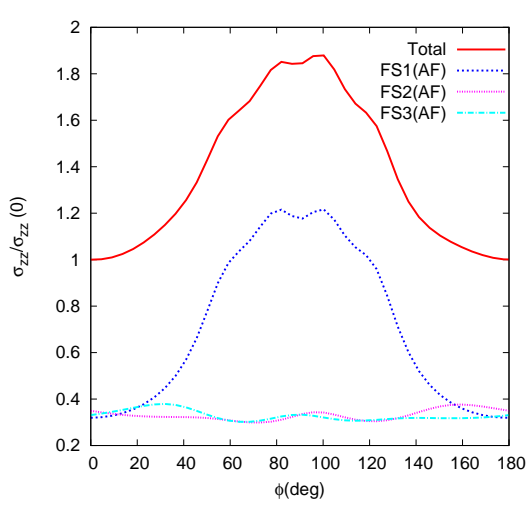

(b)

Fig. 2. (Color online) The Fermi surface of the antiferromagnetic state (a) and the normalized interlayer conductivity(b).

A clear difference between the paramagnetic state and the antiferromagnetic state is the oscillation period difference in $\sigma_{z z}$. In the paramagnetic state, the oscillation period is $90^{\circ}$. By contrast the oscillation period of the antiferromagnetic state is $180^{\circ}$. Therefore, the presence of the magnetic ordering is distinguished from the paramagnetic state by measuring the oscillation period of $\sigma_{z z}$.

In order to make clear the condition of observing the oscillations in the interlayer conductivity, we compute the magnetic field dependence of the peak height for several values of $\Gamma$. The onset of the anisotropy depends on $\Gamma$. Therefore, the measurements of the magnetic field dependence of the anisotropy provides information about the scattering. Figure 3(a) shows the magnetic field dependence of the ratio of the peak in $\sigma_{z z}$ at $45^{\circ}$ and the value at $0^{\circ}$ in the paramagnetic state. The ratio increases monotonically as we increase the magnetic field. Figure 3(b) shows the magnetic field dependence of the ratio of the peak at $90^{\circ}$ and the dip at $75.7^{\circ}$. Since the magnetic field effect on those angles are not simple, the ratio shows a non-monotonic behavior. In principle one can deduce $\Gamma$ by measuring these two ratios by comparing 
J. Phys. Soc. Jpn.

Full PAPeR

the theoretical prediction based on first principles calculations with the experiments. Figure 4 shows the magnetic field dependence of the ratio of the peak in $\sigma_{z z}$ at $90^{\circ}$ and the value at $0^{\circ}$ in the magnetically ordered state.

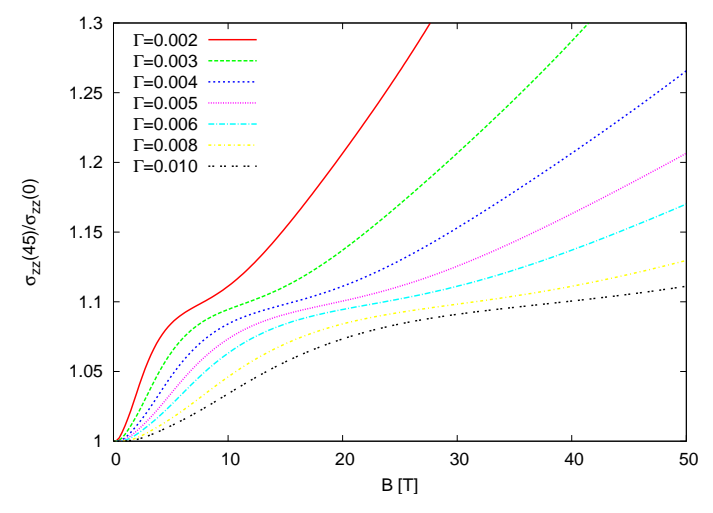

(a)

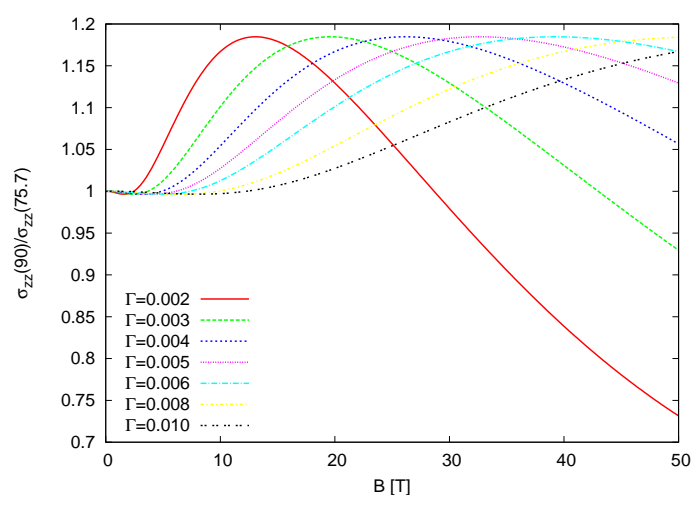

(b)

Fig. 3. (Color online) Magnetic field dependence of $\sigma_{z z}\left(45^{\circ}\right) / \sigma_{z z}\left(0^{\circ}\right)(\mathrm{a})$ and $\sigma_{z z}\left(90^{\circ}\right) / \sigma_{z z}\left(75.7^{\circ}\right)(\mathrm{b})$ in the paramagnetic state.

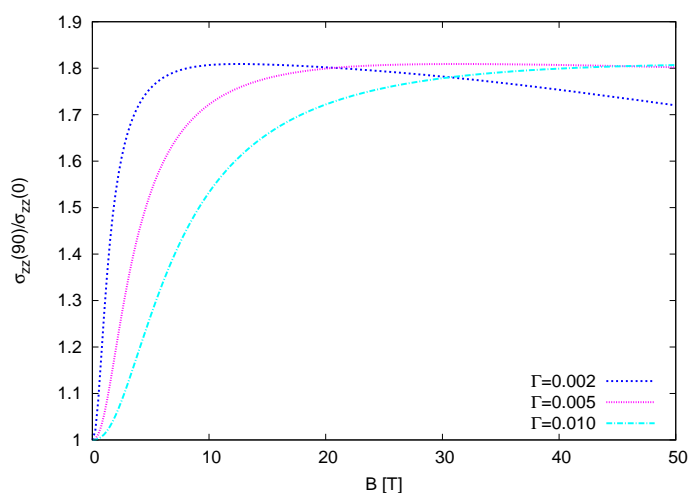

Fig. 4. (Color online) Magnetic field dependence of $\sigma_{z z}\left(90^{\circ}\right) / \sigma_{z z}\left(0^{\circ}\right)$ in the magnetic state.

\section{Doped Systems}

In this section we consider the doping dependence of the interlayer conductivity. Our consideration is based on the rigid band picture, and we take the five-band model Hamiltonian from Kuroki et al. ${ }^{23}$ Figure 5 shows the electron density dependence of the Fermi surface and Fig.6 shows the interlayer conductivity for each electron concentration with $B=10 \mathrm{~T}$ and $\Gamma=2 \times 10^{-3}$. Note that the azimuthal angle $\phi$ in Fig. 6 is shifted by $45^{\circ}$ compared to Fig. 1 because of the difference of the unit cells. (In our first principles calculations, the unit cell contains two Fe atoms and two As atoms. Meanwhile in the five band model of 
J. Phys. Soc. Jpn.

Full PAPER

ref.23, the unit cell contains one Fe atom. The Fe-Fe bond direction differs by $45^{\circ}$ between the two unit cells.) If we compare Fig.6(b) with Fig.1(b), they are qualitatively the same. The peaks at $\phi=45^{\circ}$ and $\phi=135^{\circ}$ in Fig.1(b) are observed at $\phi=0^{\circ}, 90^{\circ}$, and $180^{\circ}$ in Fig.6(b). The peak at $\phi=90^{\circ}$ in Fig.1(b) is observed at $\phi=45^{\circ}$ and $\phi=135^{\circ}$ in Fig.6(b). However, the peak widths are different. The discrepancy is mainly associated with the Fermi surface shape difference between FS4 and FS5 in Fig.1(a) and FS4 and FS5 in Fig.5(b). In addition there are non-negligible discrepancies in the Fermi velocities between the five band model and the first principles calculation. Those discrepancies lead to the differences of each band's weight in the interlayer conductivity. The Fermi velocities are the derivatives of the dispersion energies with respect to $k_{x}$ or $k_{y}$. However, we may not expect that the five band model should reproduce derivatives of the energy dispersions of the first principles calculation. Although there is some quantitative differences, the five band model reproduces the first principles result qualitatively.

In hole doped case, the result of the interlayer conductivity is similar to that of $n=6$ : For $10 \%$ hole doping case $(n=5.9)$ the Fermi surface Fig.5(a) is similar to $n=6$ case (Fig.5(b)) and so their interlayer conductivities are similar (Figs.6(a) and 6(b)). Upon further hole doping, the electron Fermi surfaces FS4 and FS5 shrink. But the shrink of those Fermi surfaces does not lead to qualitative difference of the interlayer conductivity.

In the electron doped side, for $n=6.2$, the hole Fermi surface around $(\pi, \pi)$ (FS3) disappears (Fig.5(c)). The effect of this disappearance of the Fermi surface FS3 is clearly seen in the interlayer conductivity Fig.6(c): The peak at $\phi=45^{\circ}$ and $\phi=135^{\circ}$ in Figs.6(a) and 6(b), which is associated with the flat regions of the Fermi surface FS3, vanishes in Fig.6(c). For $n=6.4$ the two hole Fermi surfaces around $(0,0)$ (FS1 and FS2) disappear, and the peak at $\phi=90^{\circ}$ in the interlayer conductivity becomes sharp (Fig.6(d)) because of large flat regions of FS4 and FS5 (Fig.5(d)).

\section{Summary and Discussion}

In this paper, we have proposed a interlayer conductivity formula under an in-plane magnetic field to study the Fermi surface topology of layered multiband systems. Applications of the formula to one of the FeAs-based superconductors, $\mathrm{LaFeAsO}_{1-x} \mathrm{~F}_{x}$, have been discussed. Although the total interlayer conductivity is given by the sum of all band contributions, it is possible to extract information about the Fermi surface topology from the peak positions in the interlayer conductivity as a function of the azimuthal angle of the magnetic field. Flat regions of the Fermi surface give rise to peaks in the interlayer conductivity, while nearly circular Fermi surfaces do not show oscillating patterns. We have discussed the difference between the paramagnetic state and the magnetic state of the parent compound LaFeAsO. As for the presence of the magnetic state, the oscillation period is $180^{\circ}$ for the magnetic state but $90^{\circ}$ for paramagnetic states. To study the doping effect, we have taken the five band model of ref.23 and discussed the Fermi surface topology change upon doping and how such changes are reflected to the interlayer conductivity.

In the analysis above, the interlayer coupling has been taken into account through the interlayer current. The energy dispersion along the $z$ axis is not included. If the electron band dispersion along the $z$ axis is 


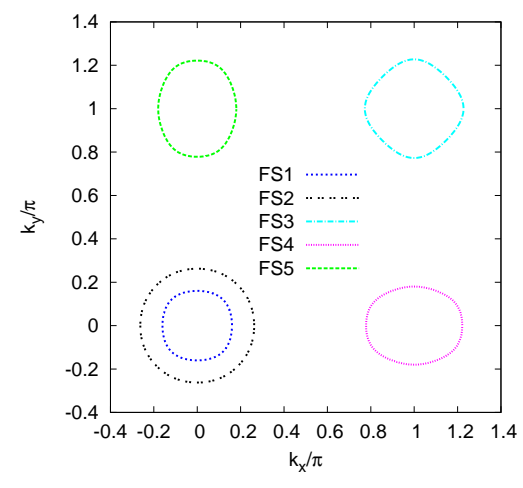

(a) $n=5.9$

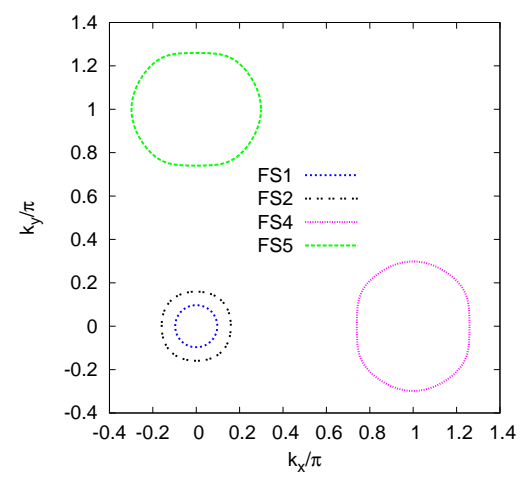

(c) $n=6.2$

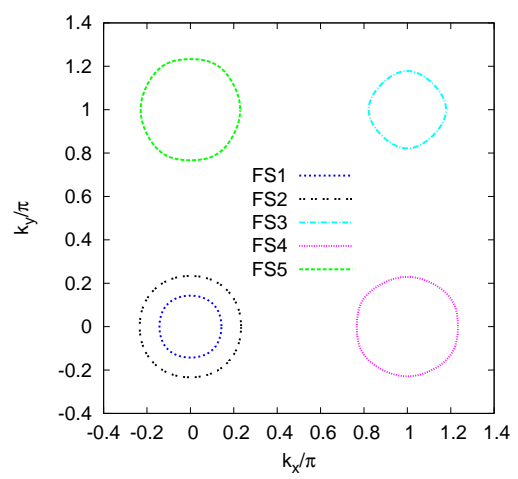

(b) $n=6$

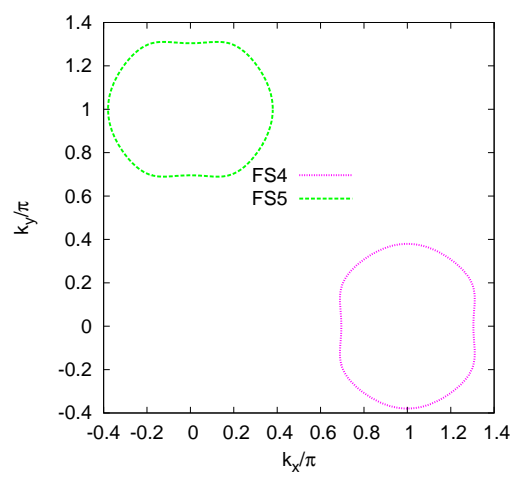

(d) $n=6.4$

Fig. 5. (Color online) Electron density dependence of the Fermi surface calculated from the five-band model by Kuroki et $a .^{23}$

simply represented by an additional term of $\epsilon_{k}^{(c)}=-2 t_{c} \cos k_{z}$, then the Lorentz function factor in eq.(10) has dominant contributions at $k_{z}=0, \pi$. However, if the Fermi energy is much larger than $t_{c}$, the Fermi surface topology change is negligible. For such a simple energy dispersion form in the $k_{z}$-direction, one may apply the Shockley's tube-integral formula ${ }^{26}$ to analyze the angle-dependent magnetoresistance oscillations. The angle-dependent magnetoresistance peaks are connected with the vanishing of the electronic group velocity perpendicular to the layers. ${ }^{17}$ Since the vanishing condition depends on the Fermi wave-vector, which is azimuthal angle dependent, it is possible to extract further information about the Fermi surface topology. ${ }^{27}$

As for the scattering, we have assumed the same scattering time for all bands for simplicity. However, the scattering time could be different for each band. In this sense, the measurement of the interlayer magnetoresistance is useful for the determination of the scattering time, because oscillating patterns are discernible from a certain magnetic field. Although the onset is not directly related to $\Gamma$, one can extract $\Gamma$ by comparing first principles calculation results with the experiments.

The results above have been obtained at zero temperature. In order to evaluate the finite temperature effect precisely, we need to sum over contributions from energy contours with including the first derivative 


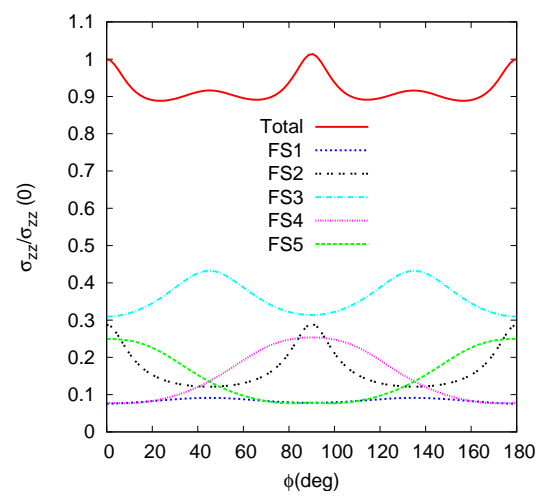

(a) $n=5.9$

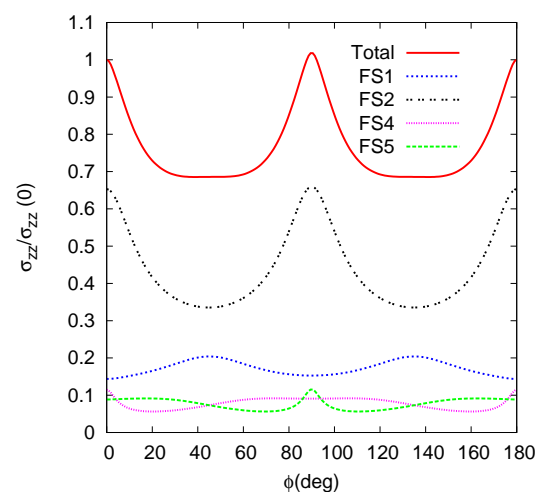

(c) $n=6.2$

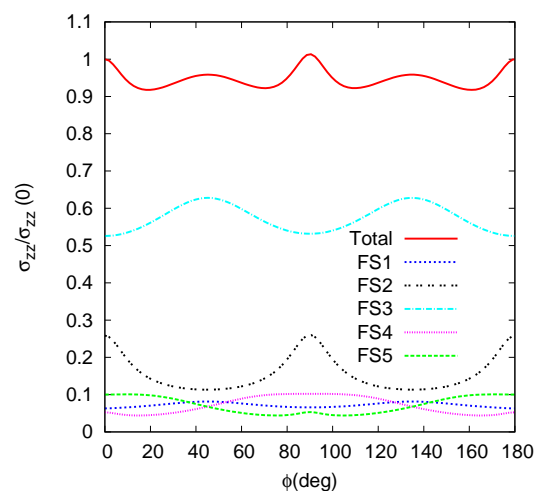

(b) $n=6$

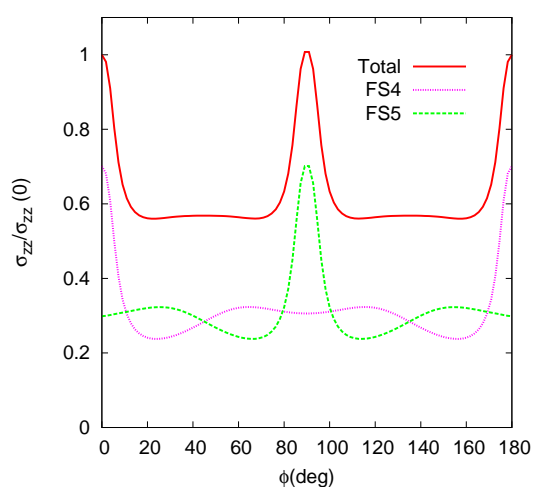

(d) $n=6.4$

Fig. 6. (Color online) Electron density dependence of the Fermi surface calculated from the five-band model by Kuroki et $a .^{23}$

of the Fermi-Dirac distribution function with respect to the energy. The finite temperature effect can be estimated for a simple energy dispersion case. If the energy dispersion is well approximated by $\epsilon_{k}=\hbar^{2}\left(k_{x}^{2}+\right.$ $\left.\lambda k_{y}^{2}\right) /(2 m)$, then the amplitude of the conductivity is somewhat suppressed for finite temperatures but the characteristic temperature for the suppression is scaled by the Fermi energy. Furthermore, the temperature dependent factor is factorized out from the magnetic field dependent part. For energy dispersions with complicated $k_{x}$ and $k_{y}$ dependences, this simple argument does not apply. However, we expect that the finite temperature effect on $\Gamma$ is more significant than that on the other contributions in the interlayer conductivity.

The interlayer magnetoresistance measurement can be used to find nodes in the superconducting state if there are nodes in the gap as discussed by Bulaevskii et al. ${ }^{28}$ If the $z$ axis current exceeds the Josephson critical current, then one can measure the interlayer magnetoresistance. In the presence of the gap nodes, the interlayer magnetoresitance detects the nodes if the applied magnetic field is parallel to the normal state Fermi velocity at the node. However, the formula given in ref.28 is not directly applicable to the $s \pm$-wave gap proposed for FeAs-based superconductivity. ${ }^{23,29}$

Finally we comment on advantages of using the interlayer magnetoresistance for investigating Fermi 
surface topology. Although ARPES is a powerful measurement to extract information about Fermi surface topology, the interlayer magnetoresistance experiments are applicable to more compounds than ARPES, since it is not necessary to cleave a sample to obtain a clean surface. In addition, the interlayer magnetoresistance is associated with the bulk properties. Therefore, the measurement is not sensitive to the surface condition. There are now many FeAs-based superconductors. In order to make clear common underlying electronic properties for the mechanism of superconductivity, we need to compare two different systems. In this sense, the interlayer magnetoresistance is useful because of its wide applicability.

We would like to thank T. Shibauchi for drawing our attention to ref.28. This work was supported by the Grant-in-Aid for Scientific Research from the Ministry of Education, Culture, Sports, Science and Technology (MEXT) of Japan, the Global COE Program "The Next Generation of Physics, Spun from Universality and Emergence," and Yukawa International Program for Quark-Hadron Sciences at YITP. 
J. Phys. Soc. Jpn.

Full PAPeR

\section{References}

1) Y. Kamihara, T. Watanabe, M. Hirano, and H. Hosono: J. Am. Chem. Soc. 130 (2008) 3296.

2) D. J. Singh and M. H. Du: Phys. Rev. Lett. 100 (2008) 237003.

3) S. Ishibashi, K. Terakura, and H. Hosono: J. Phys. Soc. Jpn. 77 (2008) 053709.

4) V. Zabolotnyy, D. Inosov, D. Evtushinsky, A. Koitzsch, A. Kordyuk, G. Sun, J. Park, D. Haug, V. Hinkov, A. Boris, C. Lin, M. Knupfer, A. Yaresko, B. Büchner, A. Varykhalov, R. Follath, and S. Borisenko: Nature $\mathbf{4 5 7}$ (2009) 569.

5) D. Lu, M. Yi, S. Mo, A. Erickson, J. Analytis, J. Chu, D. Singh, Z. Hussain, T. Geballe, I. Fisher, and Z. Shen: Nature 455 (2008) 81.

6) C. Liu, G. D. Samolyuk, Y. Lee, N. Ni, T. Kondo, A. F. Santander-Syro, S. L. Bud'ko, J. L. McChesney, E. Rotenberg, T. Valla, A. V. Fedorov, P. C. Canfield, B. N. Harmon, and A. Kaminski: Phys. Rev. Lett. 101 (2008) 177005.

7) H. Ding, P. Richard, K. Nakayama, K. Sugawara, T. Arakane, Y. Sekiba, A. Takayama, S. Souma, T. Sato, T. Takahashi, Z. Wang, X. Dai, Z. Fang, G. Chen, J. Luo, and N. Wang: Europhys. Lett 83 (2008) 47001.

8) T. Kondo, A. F. Santander-Syro, O. Copie, C. Liu, M. E. Tillman, E. D. Mun, J. Schmalian, S. L. Bud'ko, M. A. Tanatar, P. C. Canfield, and A. Kaminski: Phys. Rev. Lett. 101 (2008) 147003.

9) C. Liu, T. Kondo, M. Tillman, R. Gordon, G. Samolyuk, Y. Lee, C. Martin, J. McChesney, S. Bud'ko, M. Tanatar, E. Rotenberg, P. Canfield, R. Prozorov, B. Harmon, and A. Kaminski: cond-mat/0806.2147.

10) C. de la Cruz, Q. Huang, J. Lynn, J. Li, W. Ii, J. Zarestky, H. Mook, G. Chen, J. Luo, N. Wang, and P. Dai: Nature 453 (2008) 899.

11) Q. Huang, Y. Qiu, W. Bao, M. A. Green, J. W. Lynn, Y. C. Gasparovic, T. Wu, G. Wu, and X. H. Chen: Phys. Rev. Lett. 101 (2008) 257003.

12) A. I. Coldea, J. D. Fletcher, A. Carrington, J. G. Analytis, A. F. Bangura, J.-H. Chu, A. S. Erickson, I. R. Fisher, N. E. Hussey, and R. D. McDonald: Phys. Rev. Lett. 101 (2008) 216402.

13) H. Sugawara, R. Settai, Y. Doi, H. Muranaka, K. Katayama, H. Yamagami, and Y. Onuki: J. Phys. Soc. Jpn. 77 (2008) 113711.

14) S. Sebastian, J. Gillett, N. Harrison, P. Lau, D. Singh, C. Mielke, and G. Lonzarich: J. Phys.: Condens. Matter 20 (2008) 422203.

15) M. Kartsovnik, P. Kononovich, V. Laukhin, and I. Shchegolev: JETP Lett. 48 (1988) 541.

16) K. Kajita, Y. Nishio, T. Takahashi, W. Sasaki, R. Kato, H. Kobayashi, A. Kobayashi, and Y. Iye: Solid State Commun. 70 (1989) 1189.

17) K. Yamaji: J. Phys. Soc. Jpn. 58 (1989) 1520.

18) R. Yagi, Y. Iye, T. Osada, and S. Kagoshima: J. Phys. Soc. Jpn. 59 (1990) 3069.

19) A. G. Lebed and N. N. Bagmet: Phys. Rev. B 55 (1997) R8654.

20) A. J. Schofield and J. R. Cooper: Phys. Rev. B 62 (2000) 10779.

21) A. Drăgulescu, V. M. Yakovenko, and D. J. Singh: Phys. Rev. B 60 (1999) 6312.

22) N. E. Hussey, J. R. Cooper, J. M. Wheatley, I. R. Fisher, A. Carrington, A. P. Mackenzie, C. T. Lin, and O. Milat: Phys. Rev. Lett. 76 (1996) 122.

23) K. Kuroki, S. Onari, R. Arita, H. Usui, Y. Tanaka, H. Kontani, and H. Aoki: Phys. Rev. Lett. 101 (2008) 087004.

24) G. Mahan: Many-Particle Physics (Plenum publishers, New York, 2000) 3rd ed., pp. 505-508.

25) G. Kresse and J. Hafner: Phys. Rev. B 47 (1993) R558; G. Kresse and J. Furthmüller: Comput. Mat. Sci. 6 (1996) 15; G. Kresse and J. Furthmüller: Phys. Rev. B 54 (1996) 11169. 
J. Phys. Soc. Jpn.

FULL PAPER

26) J. Ziman: Principles of the Theory of Solids (Cambridge Univ. Press, London, 1979) 2nd ed., p. 301.

27) M. S. Nam, S. J. Blundell, A. Ardavan, J. A. Symington, and J. Singleton: J. Phys.: Condens. Matter 13 (2001) 2271.

28) L. N. Bulaevskii, M. J. Graf, and M. P. Maley: Phys. Rev. Lett. 83 (1999) 388.

29) I. I. Mazin, D. J. Singh, M. D. Johannes, and M. H. Du: Phys. Rev. Lett. 101 (2008) 057003. 